Theorie in Einklang zu setzen. Denn nach den Untersuchungen von $\mathrm{W} . \mathrm{Fre} \mathrm{i}^{2 \mathrm{I}}$ ) vermehren die Anionen $\mathrm{SO}_{4}, \mathrm{Cl}, \mathrm{NO}_{3}$ die Oberflächenspannung neutraler Gelatinelösungen in genau derselben, die Kationen $\mathrm{Na}, \mathrm{Ka}, \mathrm{Mg}, \mathrm{Ca}$ beinahe in derselben Reihenfolge, in welcher sie die Oberflächenspannung des reinen Wassers verinehren. Im Gegensatz zur inneren Reibung und zur Oberflächenspannung wird dagegen dieKo m pressibilitä t mit steigendem Kolloidfallungsvermögen mehr und mehr verringert; auch diese Veränderung dürfte in der mit der Fallungstendenz steigenden Schrumpfung (siehe oben) des Kolloids eine greifbare Erklärung fmden können. In gleicher Weise aber läBt sich verstehen, daB die Löslichkeit mit der Fallungskraft der lonen abnimmt; man weiB, daB das Lösungsvermögen irgendeiner Phase um so größer ist, je disperser ihre Zerteilung. Nur das angegebene Verhalten der lonen bei der Steigerung der Katalyse will sich der Kolloidtheorie nicht einfügen; denn es ist unwahrscheinlich, dab die Katalyse entgegen dem Dispersitätsgrade des Kolloíds eine Steigerung erfahren sollte. Aber die Ausnahme ist nur scheinbar. Denn die Verhältnisse sina nicht direkt vergleichbar, weil die Katalysen (Rohrzuckerinversion und Esterspaltung durch Säuren) in saurer, statt in neutraler Lösung beobachtet sind. Es könnte sich sehr wohl bei dieser Ausnahme nur um die bekannte Umkehrung der Hofmeister'schen Reihe bei saurer Reaktion handeln, so daß die inverse Stellung der lonen in vorzüglicher Weise der Kolloidtheorie gerecht wird. Die Ueberein-

21) Zitiert nach Wo. Ostwald, 1. c. 214. stimmung zwischen Theorie und Betrachtung geht aber noch weiter. Denn bei der Katalyse in alkalischer Losung, wo die Reihe Nitrat $<\mathrm{J}<\mathrm{Br}<\mathrm{Cl}<$ Sulfat wiederum ebenso wie in neutralem Wasser gilt, zeigt auch die lonenbeeinflussung der Katalyse ${ }^{22}$ ) den theoretisch zu fordernden Anstieg parallel zur Dispersität der ( hypothetischen) kolloiden Phase des Wassers.

Dieses Material möge genügen, um in den Hauptzägen die "Fruchtbarkeit" der kolloidchemischen Konstitutionstheorie des Wassers dafzutun. Die Theorie ist einfach, in sich einheitlich; sie hat zudem den Vorzug, experimentell nachprüfbar und im günstigen Falle beweisbar zu sein. Wenn sie sich bestätigt, wird es leicht sein, noch nach weiteren Richtungen hin ihre Folgerungen zu ziehen. Vorerst aber erfordert die Aufgabe des experimentellen Beweises neue Versuche, unter denen mir das systematische Studium der Farbe des Wassers bei Temperaturvariation und bei lonenbeeinflussung sowic die Prüfung der Umkehrbarkeit der lonenreihen bei saurer Reaktion hinsichtlich der Einwirkung auf Löslichkeit, Kompressibilität, Oberflächenspannung und innere Reibung besonders aussichtsreich erscheinen. Vielleicht aber ist es sugar zu hoffen, bei niederen Temperaturen den Kolloidcharakter des Wassers direkt der Beobachtung zugängig zu erhalten.

Leider sind mir aus Mangel an geeigneten Arbeitsbedingungen eigene Untersuchungen in diesen Fragen zurzeit nicht möglich.

22) Vgl. R. Hober, Zeitschr. f. physik. Chem. 70, 144 (1909).

\title{
Ueber die Umwandlung amorpher Körper in kristallinische.
}

\author{
Von C. Doelter.
}

(Eingegangen am 25. Màn 1910)

In einer Reihe von Aufsätzen, die in dieser Zeitschrift ${ }^{1}$ ) erschienen sind, hat P. P. vo $\mathrm{n} W$ eimar $\mathbf{n}$ den Beweis zu liefern gesucht, dab allein der kristalline Zustand der Materie zukommt, und daß eigentliche amorphe Körper nicht existieren.

Ich will nun diesen Gegenstand von etwas anderen Gesichtspunkten ausgehend betrachten. Bei Arbeiten über die künstliche Darstellung von Mineralien kam ich auf das Problem der

1) Koll.-Zeitschr. 3, 4, 5 (1908-1909).
Umwandlung amorpher Phasen in kristalline. Es ist dies die Basis der mineralogischen Synthese, bei welcher wir nicht nur die gleiche chemische Zusammensetzung des darzustellenden Minerals anzustreben haben,sondern vollkominene Identität aller Eigenschaften mit der des Naturkörpers. Als eine vollkommen gelungene mineralogische Synthese ist die des Rubins zu betrachten, da wir solche Rubine herstellen können, die von den natürichen nicht zu unterscheiden sind. Ueber die näheren Bedingungen der I mineralogischen Synthese habe ich schon vor 
langer Zeit in meiner 1890 erschienenen chemischen Mineralogie aufmerksam gemacht.

Bei den von mir lieuerdings ausgeführten Versuchen handelt es sich um die Erforschung der Umwandlung der bei Reaktionen im Laboratorium so häufig auftretenden anorphen Niederschiage in Kristalle und um die Frage, warum in der Natur chemische Verbindungen fast immer kristallinisch auftreten und amorphe homogene chemische Verbindungen so selten sind.

Nur in selteneren Fällen, nämlich bei Schmelzen, führt die chemische Reaktion zur kristallinen Abscheidung, obgleich auch da der Fall der glasigen Abscheidung eintreten kain ; bei Mineralbildung durch Abscheidung aus wässerigen Lösungen erhält man zumeist (obgleich es auch hier Ausnahmen gibt), kolloide Körper, und diese müssen durch einen besonderen Prozeb in kristalline Modifikationen umgewandelt werden.

In der anorganischen Natur ist der stabile Zustand der kristalline, und obgleich schon seit vielen Jahren auch Mineralgele bekannt waren (die Mineralogen nannten sie porodine Körper), so waren sie bis vor kurzem fast ganz vergessen.

F. Cor $\mathrm{n} u$ hat vor ganz kurzem in dieser Zeitschrift ${ }^{2}$ ) eine größere Zahl von Mineralgelen beschrieben. Allerdings sind viele Mineralgele adsorptionsartige Körper; alle anderen haben aber eine mehr oder weniger grobe Tendenz zum Kristallinwerden. Was die Masse der Mineralien anbelangt, die die Erdkruste bilden, so sind die Gele ein verschwindend geringer Anteil davon ${ }^{3}$.

Ein großer Fortschritt in der Frage nach dem amorphen. und kristallinen Zustand wurde durch die Arbeiten P.P. von We i marn's hervorgerufen.

P. P. von Weimarn zeigte den EinfluB der Konzentration auf die kristalline Auşbildung; bei mittleren Konzentrationen entstehen deutliche Kristalle, bei extremen Konzentrationen sinken solche unter ultramikroskopische Dimensionen. Dann wird wohl mit Recht darauf Gewicht gelegt, daf nur solche Massen eines festen Stoffes die Fähigkeit besitzen, die Uebersättigung einer Lösung aufzuheben, welche selbst kristallin sind, mit zunehmendem Dispersitätsgrade verlieren hochdisperse Sole diese Fähigkeit.

2) Koll.-Zeitschr. 4, 297 (1909).

3) Der von $\mathrm{Niedz}$ wiedz ki (Centralbl. t. Mineralogie 1910, Januarheit) geäußerten Ansicht, daß die Gele nur anhangsweise in der Mineralogie zu behandeln wären, kann ich mich nicht anschtießen.
Trotzdem meint Wo. Ostwald ${ }^{4}$, dab sich der ganz allgemeinen Annahme der Kristallinität aller festen dispersen Teile überhaupt gewisse Schwierigkeiten entgegenstellen; wir wollen später darauf zuräckkounmen.

Nun wäre aber vor allem die Frage zu behandeln, was ein amorpher Körper ist, falls amorphe Körper wirklich existieren, was ja neuerdings, wie erwahnt, von P. P. von Weimarn bezweifelt wurde. Letztere Ansicht P. P. von Weimarn's ist in anderer Form schon früher ausgesprochen worden, wie wir später sehen werden. Ich glaube, dak wir gegenwärtig noch nicht die Mittel besitzen, seine Ansicht zu beweisen, wie wir aber andererseits auch nicht in der Lage sind, sie als unrichtig darzustellen. Die Wahrscheinlichkeit, dab die amorphen Körper vielleicht in letzter Linie nichts anderes sind, als feinste Kristallaggregate ist keine geringe, und in dieser Hinsicht stimme ich P. P. von Weimarn $z u$, vorläufig können wir jedoch den Begriff amorph nicht entbehren.

Einige ältere Anschauungen über diesen Gegenstand will ich antühren. $\mathrm{DaB}$ wir von der alten Ansicht, der amorphe Zustand wäre der Normalzustand der festen Körper und der kristallisierte der Ausnahmezustand, schon lange abgekommen sind, braucht kaum erwähnt zu werden ${ }^{5}$.

Die Mineralogen unterscheiden schon längst die porodinen und die hyalinen Körper, welche zusammenfallen mit den Gelen einerseits und mit den Gläsern andererseits, welche zwei Arten nicht zusammengeworfen werden können.

Es ist vielleicht nicht ganz überflüssig darauf hinzuweisen, daß schon vor fast einem Jahrhundert die Ansicht verbreitet war, dab die festen unorganischen Körper kristallinisch ausgebildet seien, und dab die später zuerst von J. N. Fuchs amorph genannten Körper als Aggregate von sehr kleinen, aber unsichtbaren Kriställchen oder von Kristallkeimen betrachtet wurden $^{b}$ ).

Die Meinung, daß es keine wirklich amorphen Körper gebe, ist auch schon in etwas anderer Form seinerzeit von Frankenheim ${ }^{7}$ ) geăubert worden, welcher sagt: "Im strengen Sinne gibt es keine amorphen Körper als die flüssigen und gasförmigen, alle starren Körper sind kristallinisch."

40. Ostwald, GrundriB der Kolloidchemie (Dresden 1909), 121.

5) Vgi. O. L eh ma nn, Flissige Kristalle, 210.

9) Siehe das Zitat aus der Arbelt von J. N. Fuchs in O. Leh mann's Flussige Kristalte, 210.

i) Pogg. Ann. 72, 177 (1847). 
Auch F. G roth ${ }^{8}$ ) meinte, daB die amorphen Körper, z.B. Glas, wahrscheinlich kristalline Aggregate sind, die sich dem absolut amorphen Zustand mehr oder weniger nähern.

O. Lehmann sagt, daß man unter der Bezeichnung amorphe Körper ganz verschiedenartige Zustände zusammengefaßt hat, die durchaus nicht zueinander passen; gallertartige Körper müssen nach ihm, da sie keine einheitlichen festen Körper sind, sondern schwammige Gerüste eines festen entweder kristallisierten oder amorphen Niederschlages, dessen Poren von flüssiger Substanz erfüllt sind, ausgeschieden werden. $O$. Lehman $n$ beruft sich auch auf die Untersuchungen von $\mathrm{O}$. Bütschli, der die Wabenstruktur nachwies, sowie auch auf G. Quincke, der die Schwammstruktur der Kieselgallerten verteidigt, und auf J. M. van B e mme le ${ }^{11}$,, welcher sagt, daß die Gele im ersten Moment ihrer Bildung als Schäume aus zwei Flüssigkeiten von verschiedener Viskosität zu betrachten sind. Fr. Rin ne $\mathrm{e}^{12}$ ) erwähnt auch, dah das Gerüst der festen Partikelchen von Kieselsäuregallerte infolge der richtenden Wirkung von Kristallmolekülen, speziell bei den Zeolithen, sogar regelmäßige Struktur annehmen kann.

Bei Gläsern hat schon $G$. $Q u$ incke ${ }^{13}$ ) behauptet. dab ihnen wie auch den Kristallen Schaumstruktur zuzuschreiben ist; er beruft sich auch auf die Diffusion des Chlorsilbers durch Glas.

Nach $O$. Le h m a n $n$ ist amorphe Erstarrung Bildung einer übersättigien Lösung des festen Körpers im flüssigen.

O. N. Witt macht darauf aufmerksam, daB das Quarzglas nicht mit dem kristallisierten Quarz identisch ist. Die Glasser sind Substanzen in einem Zustande dauernder Ueberschmelzung ${ }^{14}$ ).

Quincke gelang es in älterem Aventuringlas durch Beleuchtung mit Auerlicht oder Sonnenlicht neue Kupferkristalle von $0,06 \mathrm{~mm}$ hervorzurufen.

V. Gold schmid ${ }^{15}$ ) glaubt, daB die amorphen Körper kryptomorphe seien, während W. Retgers von keinem Uebergang etwas wissen will.

$W$. Nern s ${ }^{16}$ ) nimmt mit G. Ta $\mathbf{m} \mathbf{m}$ a $\mathbf{n}$ den amorphen Zustand an, für den auch das Fehlen

5) Festrede in Kgl. Bayr. Ak., 28. Marz 1888.

9) Untersuchungent über Strakturen (Leipzig 1898).

10) Ann. d. Phys. 7, 631, 701; 9, 807 (1892).

in Zeitschr. f. anorgan. Chem. 18, 14 (1898); Bull.

23, 85 (1899).

18) Centralbl. f. Mineralogie $1902,595$.

10) Ann. d. Phys. 7, 933 (1902).

19) Siehe 0 . Lehmann, l. c. 226.

15) Zeitschr. f. Kristallogr. 29, 377 (1898).

10) Theor. Chemie, 5. Aufl., 81. eines Schmelzpunktes charakteristisch sei imit Unrecht nimmt er den Quarz als Beispiel eines anorphen Körpers an).

G. T a m m a $\mathrm{n} n$ hat den Unterschied zwischen dem amorphen Zustand und dem kristallinen näher studiert, es handelt sich bei seinen Beispielen meistens um Gläser, auf die ich später noch zurïckkomme.

Nach O. Lehman ${ }^{17}$ ) ist die Löslichkeit amorpher Körper im Flüssigkeitszustande unbeschränkt groß oder null. Diese können in Lösungen auch nicht weiter wachsen; ein amorpher fester Körper besitzt niemals die Eigenschaft reversibler Löslichkeit und damit die Fähigkeit zu wachsen wie der Kristall.

Dagegen meinte W. Retgers, daB ein Glas tatsächlich wachsen kann; auch $K$. S ch a u m ${ }^{18}$ ) erklärt sich gegen $O$. Lehmann, ebenso W. Ostwald 19 .

Barlow ${ }^{20}$ ) hat auf die Analogien zwischen Kristallen und Kolloiden aufmerksam gemacht, er glaubt auch, daß ein Zwischenzustand zwischen kristallisierten Körpern und Flüssigkeiten existiert, nämlich Flüssigkeiten mit regelmäBiger symmetrischer Anordnung der Moleküle.

Backhuis-Roozeboom ${ }^{21}$ ) ist dagegen der Ansicht, daB niemals ein kontinuierlicher Uebergang zwischen kristallisiert und amorph konstatiert ist, er legt besonderen Wert auf die verschiedene Orientierung der Moleküle in amorphen und kristallisierten Körpern.

$O$. Lehmann ${ }^{22}$ ) verweist auf die schwach doppelt brechenden und scheinbar isotropen Mischungen, welche augenscheinlich Uebergänge zwischen den amorphen und kristallisierten $\mathrm{Zu}$ ständen herstellen, und im Prinzip muB es gelingen bei Anwendung passender Substanzen völlig stetige Uebergänge bis zu durchaus isotropen Flüssigkeiten $\mathrm{zu}$ finden, welche auch amorph erstarren können. Er glaubt, dab es möglich sei, eine Substanz zu finden, deren flüssig-kristallinische Modifikation pit steigender Temperatur infolge Dissoziation der physikalischen Moleküle allmählich in die isotrop-flüssige übergeht. Dèrselbe Autor meint sogar, daB man die Frage aufwerfen könne, ob nicht is o trope Flüssigkeiten als kristallisiert zu betrachten seien.

Es wurde bereits betont, daB wir Glăser von Gallerten trennen müssen, man betrachtet sie

17) Flüssige Kristalle, 135.

19 Habilitationsschrift (Marburg 1904), 35.

19) Lehrb. d. allg. Chem. II, 2. Teil(Leipzig 1899), 202.

D) Zeitschr. f. Kristall. 29, 566 (1898).

ע) Heterogene oleichgewichte I, 153 (1901).

2) Flissige Kristalle, 230. 
als unterkühlte Flüssigkeiten; die meisten Gläser dürften bereits zahllose Kristallkeime enthalten, die aber sich zumeist der Beobachtung entziehen, die sog. Entglasungsprodukte sind als solche aufzufassen.

Unterkühlte Flüssigkeiten haben vielfach die Tendenz, sich in Kristalle umzuwandeln; bei den natürlichen Glasern, wie Obsidian, haben wir deutliche Entglasung, und dürften wir nicht anstehen, einen solchen amorphen Stoff für einen Körper zu halten, der unzählige kleinste Kristallkeime enthält.

Bei künstlichen Gläsern ist die Entglasung erst in ihrem Anfangsstadium, die Kristallisationsgeschwindigkeit der betreffenden Kristalle ist derartig klein, daB es viele Jahrzehnte braucht, um eine Entglasung hervorzubringen, in anderen Fällen kann aber die Geschwindigkeit beträchtlich $\operatorname{sein}^{23}$ ).

Nach O. Lehmann wäre der Hauptunterschied 2 wischen den Gallerten, die er nach dem Vorhergehenden von den Gläsern trennt, der, daß erstere plötzlich entstehen, während die letzteren allmählich sich bilden.

Auf eine Reihe von Unterschieden zwischen Gläsern urd den entsprechenden kristallinen Modifikationen hat besonders $\mathrm{G}$. T a $\mathrm{m} \mathrm{man} \mathrm{n}^{24}$ ) aufmerksam gemacht.

Sehr wichtig zur Beurteilung des amorphen Zustandes, verglichen mit dem kristallinen, ist das elektrische Verhalten.

Abegg und Seitz haben bei Alkoholen konstatiert, daß der glasig feste Zustand bezüglich der elektrischen Eigenschaften keine kontinuierliche Fortsetzung des flüssigen Zustandes darstellt, dagegen der glasige und kristallinische Zustand sich dielektrisch nicht merklich unterscheidet.

Ganz anders ist das Verhalten jedoch, wenn man bei Silikatgläsern die elektrische Leitfähigkeit untersucht und die Widerstandskurve des flüssigen und festamorphen, mit fallender oder steigender Temperatur verfolgt. Wenn ein Körper vom flüssigen in den kristallinischen Zustand übergeht, so ändert sich die Leitfähigkeit plötzbich sehr stark. Ganz anders ist es aber, wenn man vom flüssigen in den amorph-festen $\mathrm{Zu}$ stand ühcrgeht. Dieses Verhalten ist von grober Wichtigkeit, weil es sich zeigt, daB amorph-feste Gläser mit den Flüssigkeiten viel mehr verwandt sind, als mit den kristallisierten Körpern.

23) Vgl. S. 31 die Beobachtung Quincke's.

26) Kristallisieren und Schmelzen (Leipzig 1904).
Es gibt viele kristallisierte Silikate, die keinen festen Schmelzpunkt haben.

Sehr gute Beispiele sind Albit und Orthoklas, diese Körper gehen ohne festen scharfen Schmelzpunkt allmählich in eine viskose Flüssigkeit über, andererseits gehen sie aus dem schmelzflüssigen Zustancle allmählich in den amorphstarren über, wenn man die Widerstands-Temperaturen so konstruiert, daB $\frac{1}{T}$ auf der Abszissenachse aufgetragen wird und auf die Ordinatenachse der Logarithmus des Widerstandes, W, so erhält man für Körper, welche vom flüssigen Zustande in den kristallisierten übergehen, zwei getrennte Kurvenstücke, für Stoffe, die glasig erstarren, wie Albit und Orthoklas, jedoch nur eine einzige gerade Linie für den festen und den flüssigen Zustand. Es zeigt die Kontinuität beider Zustände, des amorphen und des flüssigen, im Gegensatze zu dem kristallinen.

In bezug auf lonenwanderung sind daher die amorphen Körper mit Flüssigkeiten vergleichbar, wenn auch in ersterem Zustande die Beweglichkeit eine viel geringere ist.

Die Wanderung von lonen in starren amorphen Körpern geht auch aus den Beobachtungen von $\mathrm{O}$. Le hmann ${ }^{25}$ ) über elektrische Diffusion in amorphem und kongorot gefärbtem Zucker hervor. Für die Gläser meinte Frankenheim, dab sie wie Fett und Harz doch aus immer noch sehr großen kristallinischen Teilchen bestehen ${ }^{26}$ ). W. Voigt ${ }^{27}$ ) spricht in bezug darauf von quasi-isotropen Körpern.

\section{Unterscheidung amorpher und kristalliner Körper.}

Als a morph bezeichnen wir einen homogenen Körper, der nach allen Richtungen gleichwertig ist, in welchem die verschiedenen Richtungen durch keine physikalische Eigenschaft des Körpers unterschieden werden können ${ }^{28}$ ). Ein amorpher Körper hat keine vektoriellen Eigenschaften. Es wäre daher theoretisch wenigstens leicht, amorphe Körper von kristallinen zu unterscheiden, in Wirklichkeit ist dies jedoch sehr schwierig. Aber gerade diese Unterscheidung ist für diese Fragen von der größten Wichtigkeit.

Optische Anisotropie ist natürlich zur Erkennung kristalliner Körper nicht maßgebend, da ja reguläre Kristalle optisch isotrop sind, in-

25) O. Lehmann, Die elektrischen Lichterscheinungen oder Entladurigen (Halle a. S. 1898), 121.

\%) Pogg. Ann. 72, 177 (1847).

27) Wiedemann's Ann. 38, 573 (1889).

s) Groth, Phys. Kristall, IV. Auflage, 3 (1905). 
dessen ist umgekehrt das Konstatieren optischer Anisotropie wohl genügend, um den kristallinen Zustand zu charakterisieren (mit Ausnahme der Fälle, wo Spannungsdoppelbrechung durch Pressen, wie bei Gelatine, Doppelbrechung zustande kommt, Fälle, die man als Zwangszustände bezeichnet hat).

Die Unterscheidung amorpher und kristalliner Niederschläge ist gewiß oft sehr schwierig, ja manchmal unmöglich, die sonst am besten $z u$ veru endende Methode auf Doppelbrechung läBt uns oft im Stiche; man kann solche Pulver, die keine Doppelbrechung zeigen, nicht ohne weiteres als amorphe erklären. Andererseits haben die sich aus einem Sol abscheidenden Gele oft auch nicht eine Spur von Anklängen an den kristallinen Zustand. Im übrigen spielt bei der Unterscheidung das subjektive Moment jedenfalls eine bedeutende Rolle, und was der eine als amorph bezeichnet, wird vielleicht ein anderer als kristallin benennen. Wenn Silbersole einen kristallinen Niederschlag ausscheiden, wie P. P. von Weimarn zeigt, so würde das keinesfalls auf andere Stoffe zu verallgemeinern sein.

Es ist gewib, daB manche Niederschläge bei geringen Vergrößerungen sich als scheinbar amorph erweisen, die sich dann bei den stärksten Vergrökerungen doch als kristallinische entpuppen. Es ist daher nicht ausgeschlossen, daB der amorphe Zustand zum Teil nur ein Scheinzustand ist, der aus der Unzulänglichkeit der Untersuchungsmethoden resultiert.

\section{Die Arbeiten P. P. von Weimarn's.}

P. P. von Weim arn hat das Verdienst, auf den Finflub der Konzentration der Lösung bei der Bildung kristalliner beziehungweise kolloider Niederschläge aufmerksam gemacht zu haben. Seine Studien hat er hauptsăchlich an Bariumsulfat und Aluminiumhydroxyd ausgeführt, also an anorganischen Körpern. Es wäre nötig, dieselben auf Stoffe aus der organischen Chemie zu erweitern, die ja mehr amorphe Stoffe liefert.

Bei Aluminiumhydroxyd und bei Bariumsulfat erhält man wohl anfangs auch amorphe Niederschlagge (vgl. weiter unten) aber nach den Ausführungen von P. P. von. Weimarn scheint es, daß man verhaltnismaBig rasch kristalline Niederschläge erhalt, falls man die richtigen Konzentrationen wähit.

Er meint ${ }^{29}$ ), daB das Aluminiumhydroxyd ziemlich leicht kristallisiert, und dab die Tatsache, dab es gewöhniich gallertartig erhalten

\footnotetext{
m) 1. c. 37 .
}

wird, nur davon abhängt, daß die Löslichkeit des Aluminiumhydroxyds in reinem Wasser nur gering ist.

Das Gebiet sogenannter amorpher Niederschläge ist für den genannten Körper sehr weit, es beginnt etwas höher als bei $\frac{\mathrm{N}}{500}$.

Bezüglich der $\mathrm{BaSO}_{4}$-Niederschläge sagt P. P. von Weimarn, daB das allgemeine Bild des Einflusses, den die Konzentration der reagierenden Lossungen ausübt, deutlich dafür spricht, dab beim progressiven Anwachsen der Konzentration die kristallinische Substanz progressiv feiner wird.

Der zweite indirekte Beweis wäre der, dak, wenn man einen in konzentrierten Losungen entstandenen $\mathrm{BaSO}_{4}$-Niederschlag auch für amorph hălt, er nach der Lehmann'schen Definition des amorphen Körpers nicht weiter wachsen könnte, und also auch nicht als Keim dienen kann und deshalb müßte neben einem amorphen Niederschlag stets eine genügende Menge eines kristallinen Niederschlags entsprechend dem Reaktionsverlauf in verdünnten Lösungen vorhanden $\operatorname{sein}^{30}$ ).

-P. P. von Weimarn bemerkt noch, daB er zwischen feinflockigen und gallertartigen Niederschlägen des $\mathrm{Ba} \mathrm{SO}_{4}$ keinen einzigen deutlichen Mikrokristall bemerkte.

"Die sogenannten, dem Aussehen nach amorphen Niederschläge des Bariumsulfats, haben noch ihren kristallinen Charakter; die der Kugel nahestehende Form ist das Resultat optischer Begleiterscheinungen, die das mikroskopische Bild verwischen." Dies wäre, falls es sich allgemein bestätigen würde, allerdings geeignet, die P. P. von We i$m$ a rn'sche Ansicht in Gänze zu adoptieren. Indessen sind wir jedoch noch einigermaben davon entfernt, diesen Satz als bewiesen anzunehmen, wie die Untersuchung vieler Gele bei starken Vergrößerungen zeigt.

Ein amorphèr Körper kann also nur scheinbar amorph sein, und dies ist ein Fall, der häufig zutrifft; hier scheint nun von Weimarn im Rechte zu sein und seine Ausführungen, daB ein amorpher Körper nur scheinbar amorph ist, bei sehr starker Vergrößerung aber sich als kristallin erweist, wie bei $\mathrm{Al}_{2} \mathrm{O}_{3} \cdot 3 \mathrm{H}_{2} \mathrm{O}$, verdienen große Beachtung, wenn sie auch zurzeit nicht verallgemeinert werden dürfen. Man hat, wie erwähnt, viel Gewicht auf die optischen Eigenschaften bei der Entscheidung, ob kristallin

\footnotetext{
๑) $\$ 7,5296$.
} 
oder amorph, gelegt, und in der Tat gelingt damit die Unterscheidung sehr gut in manchen; aber weitaus nicht in allen Fallen; diese sehr dünnen und kleinen Kriställchen zeigen keine Doppelbrechung, unsere Hilfsmittel reichen dann nicht mehr aus.

P. P. von Weimarn stützt sich sehr auf die Definition von $O$. Leh ma $n$ Körper nicht wachsen können, aber er übersieht, da日, wenn es keine amorphen Körper gibt, dann auch diese Unterscheidung wegfallen müBte; das Wachstum eines amorphen Körpers wird jedenfalls nicht ganz mit dem eines kristallisierten Körpers zusammenfallen und in der Theorie auch verschieden sein, die Betrachtung von Hyalith und Opal zeigt aber doch, daB ein Wachstum mőglich ist. Und bei typischen Gelen habe ich ein allmăhliches Zusammenballen, eine Vergrößerung beobachtet, die doch auch ein Wachstum ist, wenn auch von etwas verschiedener Art. Jedenfalls kann man die Definition des Kristalls von $\mathrm{O}$. L e $\mathrm{h}$ m a $\mathrm{n} n$. nicht dazu benutzen, um umgekehrt daraus $2 u$ schlieben, dab es keine amorphen Körper geben kann, wenn Wachstum beobachtet wird.

Allerdings ist es immerhin wahrscheinlich, daB das Wachstum eines Gels ein anderes ist, als das eines Kristalles. Bei Gläsern erfolgt jedoch die amorphe Erstarruing in ähnlicher Weise, wie bei kristallisierten Körpern, nur dab sie nicht von einer verhaltnismäbig geringeren Zahl von Zentren ausgeht; auch andere Autoren (S. 31) sind der Ansicht, daß bei Gläsern eine Erstarrung wie bei Kristallen eintritt.

Ich gebe $\mathbf{z u}$, dab man bei vielen Körpern, die früher als amorph bezeichnet wurden, jetzt diese eher als kristallinische wird annehmen können, trotzdem ist, wenn man z. B. die Unwandlung des amorphen Niederschlages in der Schüttelmaschine oder auf dem Wasserbade verfoigt, die Umwandlung zu konstatieren. Ich glaube auch kaum, dab an dem C. P a al'schen Chlornatriumgel irgend etwas zu beobachten ist ${ }^{31}$ ), was auf kristallinen Charakter bezogen werden könnte, auch ist durch Lagern derselben keine Umwandlung zu konstatieren, und ich konnte nach mehr als 6 Monaten keine Veränderung der Tropfen beobachten.

P. P. von Weimarn hat auch bei kristallinen. Niederschlägen auf den Einfluß der ein-

a) Vgl. C. Doelter, Das Radium und die Farben (Thusdor Steinkopff, Dresden 1910). geschlossenen fremden Substanz auf den Kristallisationsprozeb aufmerksam gemacht. Die Bildung gallertartiger Niederschläge und sogar von Glas soll bedingt sein durch den Einflub fremder Substanzen auf die Vergrößerung der Anzahl der Kristallisationszentren ${ }^{32}$ ). Gläser und opalähnliche Massen sind nach ihm keine amorphen Systeme, sondern schwammähnliche Aggregate feinster Kriställchen, die von Lösungen oder Flüssigkeiten getränkt sind. Es ist das in anderer Form eine Rückkehr zur Ansicht O. L e h m a n n's, daß ein amorpher Körper eine Lösung des kristallinischen Körpers in der Flüssigkeit sei, eine Ansicht, der ein gewisser Grad von Wahrscheinlichkeit nicht abzusprechen ist.

BeimVergleiche amorpherKörper und Flüssigkeiten kommen wir zu dem Schlusse, dak amorphe wie kristalline Körper aus einer nicht kristallinen Flüssigkeit entstehen, welche wir z. B. im Falle der Gläser mit einem ganz amorphen Glase vergleichen können. Dann haben wir die polymorphen Umwandlungen verschiedener Kristallarten, bei welchen wir doch einen ähnlichen Prozeb uns vorstellen, wie bei der Umwandlung eines amorphen Körpers in einen kristallinen. Es wendet von Weimarn gegen letztere ein, daB in wirklich amorphen Körpern keine Impfung mangels Impfkristallen eintreten kann, aber wir wissen ja, das auch spontane Kristallisation aus Flüssigkeiten moglich ist.

Sehr schwer zu erklaren ist der amorphe Zustand bei Elementen, und es wăre jedenfalls eine befriedigende Losung, wenn sich $z$. B. amorpher Schwefel als ein Aggregat feinster kristallinischer Teilchen erweisen würde, denn die bisherigen Erklärungen sind recht unbefriedigend ${ }^{33}$ ).

Wir stehen im ganzen doch noch vor einer ungelosten Frage, und die Beweise dafür, dab so viele amorphe Körper es in Wirklichkeit nicht sind, sind keine direkten, sondern mehr indirekte.

Vor allem müBte, wenn es keine amorphen Körper gebe, ein Uebergang zwischen den scheinbar amorphen und den kristallinen vorkommen, direkt ist aber bis jetzt kein solcher konstatiert worden, es fehlt aber nicht an Stimmen dafür, außer den früher erwähnten haben sich auch andere Forscher dafür ausgesprochen (vgl. weiter unten S. 14).

38) 1.c. $\$ 22$.

*) Vgl. C. Doelter, Phys. chem Mineral., 4. (SchluB folgt.) 